\title{
LETTERS
}

\section{General Practice Services Committee incentives are drivers in enticing medical students to choose family medicine}

I am writing regarding the recently published CMAJ article that reviewed the British Columbia General Practice Services Committee (GPSC) Complex Care incentive, which I believe contains many misrepresentations of previous research articles about the chronic disease management incentives in BC. The authors ${ }^{1}$ state that their "findings contradict earlier claims of improved continuity and cost savings based on cross-sectional comparisons, but are generally consistent with previous research that has found limited impact of incentives within primary care."

The initial assessment of chronic disease management for diabetes and heart failure that was implemented September 2003 showed that patients eligible for the incentive became more strongly attached to their family physician. This resulted in cost avoidance owing to decreased emergency department visits and hospital admissions. The complex care incentive requires the patient to have at least two conditions from sepa- rate eligible condition categories, including diabetes and heart failure. The largest cohort of complex care patients includes those with diabetes. It is likely that most of the patients with diabetes and heart failure had already experienced an increase in "stickiness" to their general practioner as a result of the chronic disease management incentive, before the implementation of the Complex Care incentive. Since this recent paper only looked at data from 2005, it would not be able to distinguish the impact of the Complex Care incentive as a factor independent of the chronic disease management incentives introduced in 2003 . All reports can be found on the GPSC website (available at www.gpscbc.ca/our-impact/ evaluations/evaluations).

Previous evaluation of the Complex Care fee showed that, when the cost of all billable incentives was included, there was no overall cost avoidance. However, in a subgroup of patients with specific conditions (chronic obstructive pulmonary disease and heart failure in particular), there was a substantial cost avoidance even with the inclusion of the cost of GPSC incentives.

The initial mandate of the GPSC in 2002 was to support full-service family practice. Prior to the introduction of the
GPSC incentives, family medicine was the first choice of residency for only $24 \%$ of medical students, and this has risen in $\mathrm{BC}$ to over 40\% (see www.carms.ca/wp-content/ uploads/2016/08/Chart_12_Choice_of_ Family_Medicine_by_sex_1994\%E2\% 80\%932016.pdf and http://postgrad.med. ubc.ca/2015/03/04/ubc-celebrates-carms -match-day-2015/).

I believe the GPSC incentives have been a substantial driver in enticing medical students to choose family medicine as a postgraduate career.

\section{Catherine A. Clelland MD}

Family physician, New Westminster, BC

Cite as: CMAJ 2017 February 13;189:E250. doi: $10.1503 / \mathrm{cmaj} .732457$

\section{Reference}

1. Lavergne MR, Law MR, Peterson S, et al. A population-based analysis of incentive payments to primary care physicians for the care of patients with complex disease. CMAJ 2016;188:E375-83.

Competing interests: I practise in $\mathrm{BC}$, and I was the President of the Society of General Practitioners (SGP) of BC (Section of GP at the British Columbia Medical Association) in 2002-03 and Executive Director of the SGP from 2004 to 2013. I have also participated in GPSC working groups over the past 13 years. 